\title{
Molecular damping: Mechanical response of self-assembled monomolecular layer to compression
}

\author{
D. Devaprakasam and S. K. Biswas* \\ Department of Mechanical Engineering, Indian Institute of Science, Bangalore 560 012, India
}

\begin{abstract}
We use a displacement-controlled dual double-cantilever-based surface-force-type apparatus to dynamically probe perfluorooctyl trichlorosilane monolayers self-assembled on aluminum and silicon substrates of $1 \mathrm{~nm}$ and $0.7 \mathrm{~nm}$ rms (root mean square) roughness, respectively, using a $1.12 \mathrm{~mm}$ diameter ruby sphere of $0.25 \mathrm{~nm}$ rms roughness. We record stiffness and damping constant as a function of compression load and deconvolute the elastic modulus using contact mechanical formulations. When mechanical intervention is limited to the terminal end of the molecule there is a strong viscous response and a low level of elastic response in consonance with the ability of the molecule to generate conformational defects freely. When the intervention penetrates into the molecular backbone the damping disappears dramatically and the molecule registers at a contact mean pressure of about $0.2 \mathrm{GPa}$ a monotonic and steep rise in elastic resistance in response to further intervention by the probe. We offer a physical explanation of the process and describe this change as due to a phase transition from a liquidlike to a solidlike state as indicated by a large increase in relaxation time constant.
\end{abstract}

\section{INTRODUCTION}

Mechanical properties of substrate-supported organic overlayers have evoked considerable interest in engineering and biomedical disciplines. In a wide variety of practical application the overlayers are trapped between the sliding surfaces as a boundary lubricant. ${ }^{1}$ These overlayers are generally $2-3 \mathrm{~nm}$ thick chemically grafted monomolecular layers which, if efficient, support the compressive load, provide damping, and thus prevent contact of mating surfaces. This ensures continuance of smooth sliding without adhesion ${ }^{2}$ and wear. ${ }^{3}$ The latter are endemic in systems such as power transmission machinery, ${ }^{4,5}$ microelectromechanical system, ${ }^{2,5}$ and total replacement human joints. ${ }^{6,7}$ For selection of a boundary lubricant suited to an application a knowledge of the mechanical properties under compression and shear loading is essential. Surface force apparatus (SFA) is a useful instrument to determine these properties. The instrument involves large [compared to what is met in atomic force microscope studies (AFM)] contact $\operatorname{areas}^{8,9}$ to measure aggregate properties, thus averaging out the many local variations between molecules generally reflected in AFM data. SFA has been successfully used ${ }^{10,11}$ to investigate the shear response of molecules posited on solid surfaces. The response of selfassembled monolayers to compression has, however, received less attention, perhaps because of experimental difficulties. The limited work has established that these monolayers under compression kink and bend, ${ }^{12,13}$ respond as a time-dependent elastic system, ${ }^{14,15}$ and become reversibly disordered. ${ }^{16}$ In this paper we report the estimation of mechanical properties under compression of a silane-based monolayer assembled ${ }^{17}$ on aluminum. We compress the monolayer placed on a flexible platform, vibrate the pressing probe sinusoidally at subresonance frequency and subnanometric amplitude, and record the dynamic response of the platform. We determine the damping constant and Young's modulus of the self-assembled monolayer (SAM) as a function of compression load.

\section{EXPERIMENT}

Mechanical properties of thin films are measured by mounting the film on a rigid substrate and probing it with a rigid tip mounted on a stiff spring. The thinness of monolayers, however, poses a special problem. Here the depths probed have to be limited to avoid the influence of the substrate material on the measured property. ${ }^{14,15}$ Direct measurement of property is therefore problematic as a stiff system is required to provide very high displacement resolution and displacement control. The surface force apparatus ${ }^{18}$ partly addresses this problem by using optical techniques for displacement measurement but is therefore also encumbered with the use of a transparent substrate and probe to confine the molecules subjected to compression. In this paper we test monomolecular surface-active films confined between two opaque engineering surfaces. We achieve high displacement resolution by recording the displacement in the sample as the differential of displacements of two parallel dual doublecantilever systems, ${ }^{19}$ one carrying the probe and the other carrying the substrate on which the sample is self-assembled. As the substrate is now placed on a flexure we are able to control the penetration of the monolayer to subnanometric levels. We measure displacements of the two cantilevers using capacitative sensors of $0.01 \mathrm{~nm}$ resolution and obtain the force knowing the stiffness of the cantilevers. We have designed the cantilevers as integral parts of a monoblock to have a stiffness $(\approx 15000 \mathrm{~N} / \mathrm{m}$ ) (for the cantilever stiffness calibration procedure see Ref. 19) of the same order as that of the monolayer. As this stiffness, though high enough to obtain good signal-to-noise ratio, is not high enough to allow direct measurement of properties we sinusoidally modulate the probe at subresonance frequencies (natural frequency $\approx 200 \mathrm{~Hz})$ and subnanometric $(0.5 \mathrm{~nm})$ amplitude. We measure the force response and record the gain and phase difference using a lock-in amplifier. We next use a Kelvin viscoelastic model to deconvolute the stiffness and damping constant of the monolayer. If $K_{c}$ is the stiffness of the flexure 


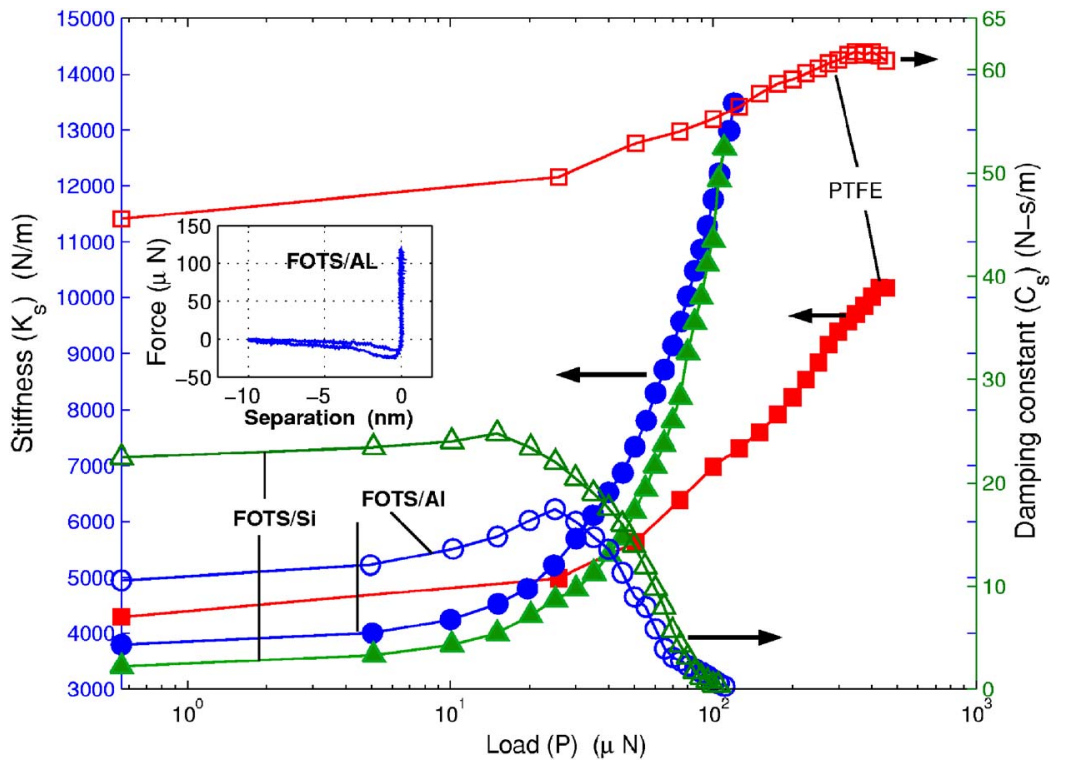

FIG. 1. (Color online) Measured stiffness and damping constant, deconvoluted using Eq. (1). The inset shows the load separation for FOTS/Al. Filled symbols, stiffness; unfilled symbols, damping constant. $\square$, PTFE; $\bigcirc$, FOTS/Al; $\triangle$, FOTS/Si. supporting the substrate, $\omega=2 \pi f$, where $f$ is the drive frequency, $R$ is the gain and $\phi$ is the phase difference, the stiffness $K_{s}$ and damping constant $C_{s}$ of the monolayer are given by. ${ }^{20}$

$$
K_{s}=\frac{K_{c}\left(R \cos \phi-R^{2}\right)}{\left(R^{2}-2 R \cos \phi+1\right)}, \quad C_{s}=\frac{\left(K_{c} R \sin \phi\right)}{\omega\left(R^{2}-2 R \cos \phi+1\right)} .
$$

A boundary lubrication additive generally has a hydrophobic terminal group, a carbon backbone, and an anchoring head group. The mechanical response of such a molecule in compression is thus likely to depend on the extent of compression. We compress the monolayer to different levels by varying the direct loading of the monolayer in contact with the probe and obtain the dynamic response of the film at different loads. In these measurements we maintain the time period of the drive signal to be significantly greater than the expected relaxation time constant of self-assembled monolayers ${ }^{14}$ to allow the molecules to fully relax at the end of a cyclic intervention and before the starting of the next. The experiments were carried out with a ruby probe of $1.12 \mathrm{~mm}$ radius and $0.25 \mathrm{~nm} \mathrm{rms}$ surface roughness and an aluminum substrate in a humidity chamber where the relative humidity was maintained at less than $10 \%$. A polycrystalline aluminum ( $99.99 \%$ purity) flat was polished to a root mean square roughness of $1 \mathrm{~nm}$, ultrasonicated in 50-50 acetonewater mixture, and dried in dry ultrapure nitrogen. $1 \mathrm{~m} M$ $1 \mathrm{H}, 1 \mathrm{H}, 2 \mathrm{H}, 2 \mathrm{H}$-perfluorooctyl trichlorosilane ${ }^{17}$ (FOTS) in isooctane was prepared and the aluminum substrate was dipped in it for $30 \mathrm{~min}$. The substrate was taken out, washed in isooctane and again dried in dry nitrogen gas. Grazingangle Fourier transform infrared spectroscopy was used to obtain spectra of the deposited film to ensure monomolecular coverage of the substrate by the SAM. A few experiments were also done on bulk polytetrafluoroethylene (PTFE) disks. A PTFE rod of $1 \mathrm{~cm}$ diameter was machined to give a disk of thickness $2 \mathrm{~mm}$; the disk was polished using a commercially available polishing cloth. The experiments on bulk
PTFE helped us to benchmark the experimental approach. The tests showed hysteresis in the force curve, indicating plasticity on contact, and yielded a storage modulus of $0.35 \mathrm{GPa}$ and a loss modulus of $65 \mathrm{MPa}$, both consistent with values reported by others. ${ }^{21}$

\section{RESULTS: MECHANICAL RESPONSE}

As the ruby sphere approached the FOTS SAM the substrate moved up over a $9 \mathrm{~nm}$ distance registering a small attractive force (Fig. 1). The difference between probe and substrate displacements is the separation, negative separation indicating a region of noncontact. Very close to zero separation the force in the repulsive region increases sharply (Fig. 1 inset). The elastic (devoid of hysteresis) behavior of the SAM seen in the repulsive part of contact loading-unloading has also been observed by others. ${ }^{14,15}$

Figure 1 shows the stiffness $K_{s}$ and the damping constant $C_{s}$ of the SAM estimated using Eq. (1), knowing the gain and phase difference recorded at a frequency of $5 \mathrm{~Hz}$ and amplitude of $0.5 \mathrm{~nm}$, as a function of normal force applied to the SAM. The increase in stiffness and damping constant commences in the attractive part of the force regime. The damping constant of the monolayers reaches a maximum for a FOTS SAM at about $30 \mu \mathrm{N}\left(\times 10^{-6}\right)$. Increasing the normal load further brings about (1) a sharp decrease in this damping constant (it becomes zero at $120 \mu \mathrm{N}$ ) and (2) a steep monotonic increase in stiffness with compression. The stiffness characteristic is perfectly reversible while the damping characteristic shows hysteresis on unloading. This behavior of the SAM is significantly different from that of a bulk polymer PTFE which exhibits a very gradual increase of its damping capacity (Fig. 1) over a large range of load. It has been suggested ${ }^{14-16}$ that at high loads, the measured mechanical properties may largely be accounted for by the mechanical property of the substrate, for sharp tips which penetrate the monolayers easily ${ }^{13,14}$ and for large radius probes where the substrate is deformed. ${ }^{13,15}$ For our experiments on the FOTS SAM these possibilities seem unlikely as even at 


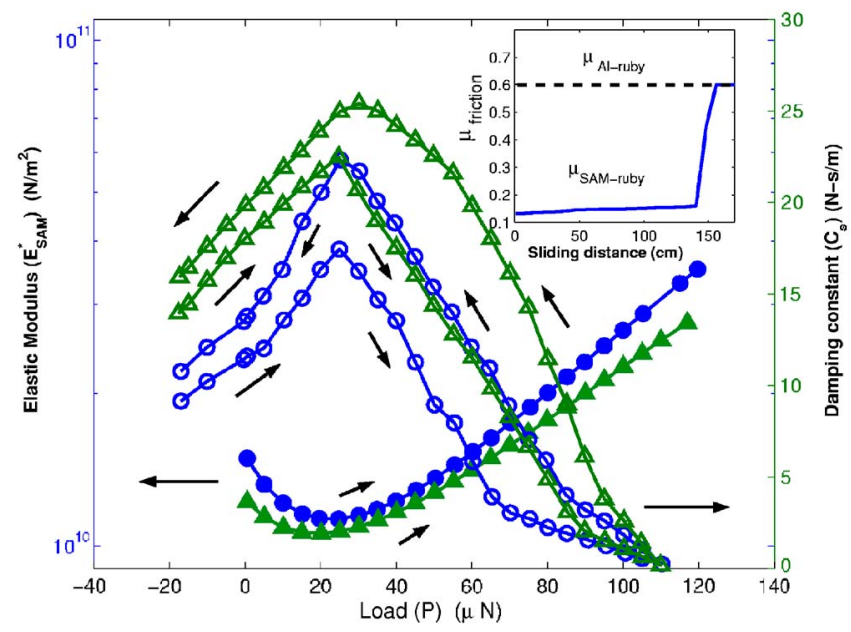

FIG. 2. (Color online) The Young's modulus deconvoluted from stiffness data (Fig. 1) using Eq. (5) and damping constant to show their opposite trends with load. The inset shows friction coefficient measured for FOTS/Al by a ruby ball under $100 \mathrm{mN}$ normal load. Filled symbols, elastic modulus; unfilled symbols, damping constant. $\mathrm{O}$, FOTS/Al; $\triangle$, FOTS/Si. Arrows show loading direction.

the highest test load $(120 \mu \mathrm{N})$ our dynamic measurements gave finite values of gain $(R)$ and phase differences $(\phi)$. We recorded for base aluminum substrate $R=1$ and $\phi=0$ and for FOTS SAM $R=0.45$ and $\phi=2$. We explored this further by probing the SAM now deposited on a silicon wafer of $0.7 \mathrm{~nm} \mathrm{rms}$ roughness. Figure 1 shows that the stiffness of FOTS/aluminum is slightly higher than that of FOTS/silicon; this slight difference is possibly due to the lower roughness of the silicon substrate (higher modulus, if the substrate material property is influential, should yield higher stiffness). We also conducted ball-on-disk experiments on FOTS SAM using a fixed ruby ball (1.12 $\mathrm{mm}$ diameter) in a Nanotribometer (CSEM, Switzerland) under $100 \mathrm{mN}$ load. Figure 2 (inset) shows that even under this heavy load (compared to the SFA experiments) the low friction characteristic of ruby sliding on a SAM is maintained up to a sliding distance of $1.4 \mathrm{~m}$. If we fit a polynomial to the stiffness characteristics (Fig. 1) it gives

$$
S=K_{1}+K_{2} P+K_{3} P^{2},
$$

where $P$ is the applied load, $K_{1}=3466 \mathrm{~N} \mathrm{~m}^{-1}, K_{2}=65.5$ $\times 10^{6} \mathrm{~m}^{-1}$, and $K_{3}=0.15 \times 10^{12} \mathrm{~N}^{-1} \mathrm{~m}^{-1}$. As the probe displacements in our compression experiments are of the same order as the surface roughness, we take roughness into account in deconvoluting the elastic modulus of the SAM. We assume that the SAM conforms to the substrate surface undulations. We take the maximum negative (attractive) load $(20 \mu \mathrm{N})$ achieved in the unloading part of the forcedisplacement characteristics (Fig. 1, inset) as an adhesion overload $\left(P_{a d}\right)$. Assuming that this overload is invariant with applied load (even if the surfaces are rough) and that the applied and the adhesive loads are additive as in the Derjaguin-Muller-Toporov model of adhesive contact, we estimate $^{22}$ the error introduced in total load because of the "invariance" assumption. The error is about $8 \%$ of the ap- plied load in the $20-120 \mu \mathrm{N}$ range. The real area of contact may be given as

$$
A_{r}=\frac{\left(P+P_{a d}\right)}{\left(k_{s} \sigma_{s}\right)^{1 / 2} E^{*}},
$$

where $k_{s}\left(\approx 10^{6} \mathrm{~m}^{-1}\right)$ is the reduced (probe and substrate) mean summit curvature and $\sigma_{s}\left(\approx 10^{-9} \mathrm{~m}\right)$ is the equivalent (probe and substrate) standard deviation of summit heights, both obtained experimentally (Thermomicroscope Explorer $\mathrm{AFM}$, Veeco Instruments, USA) and $E^{*}$ is the reduced modulus. We now express stiffness as

$$
S=2 E^{*} \sqrt{\frac{A_{r}}{\pi}} .
$$

Combining Eqs. (2)-(4) gives

$$
\begin{aligned}
E^{*}= & \frac{\pi}{4} \frac{\left(k_{s} \sigma_{s}\right)^{1 / 2}\left(K_{1}+K_{2} P+K_{3} P^{2}\right)^{2}}{\left(P+P_{a d}\right)} \\
& \text { where } \frac{1}{E_{S A M}^{*}}=\frac{1}{E^{*}}-\frac{1-\nu_{\text {probe }}^{2}}{E_{\text {probe }}} .
\end{aligned}
$$

For an $E_{\text {probe }}=270 \mathrm{GPa}, \nu_{\text {probe }}=0.15, E_{S A M}^{*}$ [Eq. (5)] is plotted in Fig. 2 and shows a modulus level which is of the same order as that reported for other SAMs. ${ }^{23}$ The high relaxation times (low phase shift) observed here suggest the alternative possibility of the responses recorded here being those of water molecules condensed or adsorbed at the hydrophilic probe end as they are swept away by probe displacement. We rule this out as improbable because the modulus recorded (of the order of $10 \mathrm{GPa}$ ) in the range of displacement where the damping is active is unlikely to correspond to that of water.

The data presented here give, at a load of $120 \mu \mathrm{N}$, a real contact area to apparent contact area ratio of 0.02. The damping and stiffness characteristics shown in Fig. 2 indicate that the mechanical response of the monolayer is load induced. In this the response is analogous to that of bulk polymers where properties such as yield are functions of the applied hydrostatic pressure. Where the response of the monolayer deviates from that of the bulk polymer is that the monolayer response to normal load is scale dependent (length scale along the molecular backbone). This nonlinear response is due to the fact that constraint to molecular motion varies along the length of the molecule. At the terminal end the molecule enjoys a large degree of freedom; going toward the substrate such freedom of motion is prevented by the highly constrained volume.

We explore this by making an estimate of the compression (displacement) of the monolayer as a function of applied load. This is now possible ${ }^{22}$ known the modulus (Fig. 2) and the adhesive interaction energy between the monolayer and the ruby probe. The latter we determine experimentally by contact angle measurement ${ }^{24}$ as $22 \mathrm{~mJ} / \mathrm{m}^{2}$. Assuming that the contact commences at a distance of $\sigma_{s}$ from the mean roughness line we determine the displacement of the probe as a function of applied load. A strict one-to-one correlation cannot be made between the estimated displacement of the probe and distances from the terminal end of a single mol- 


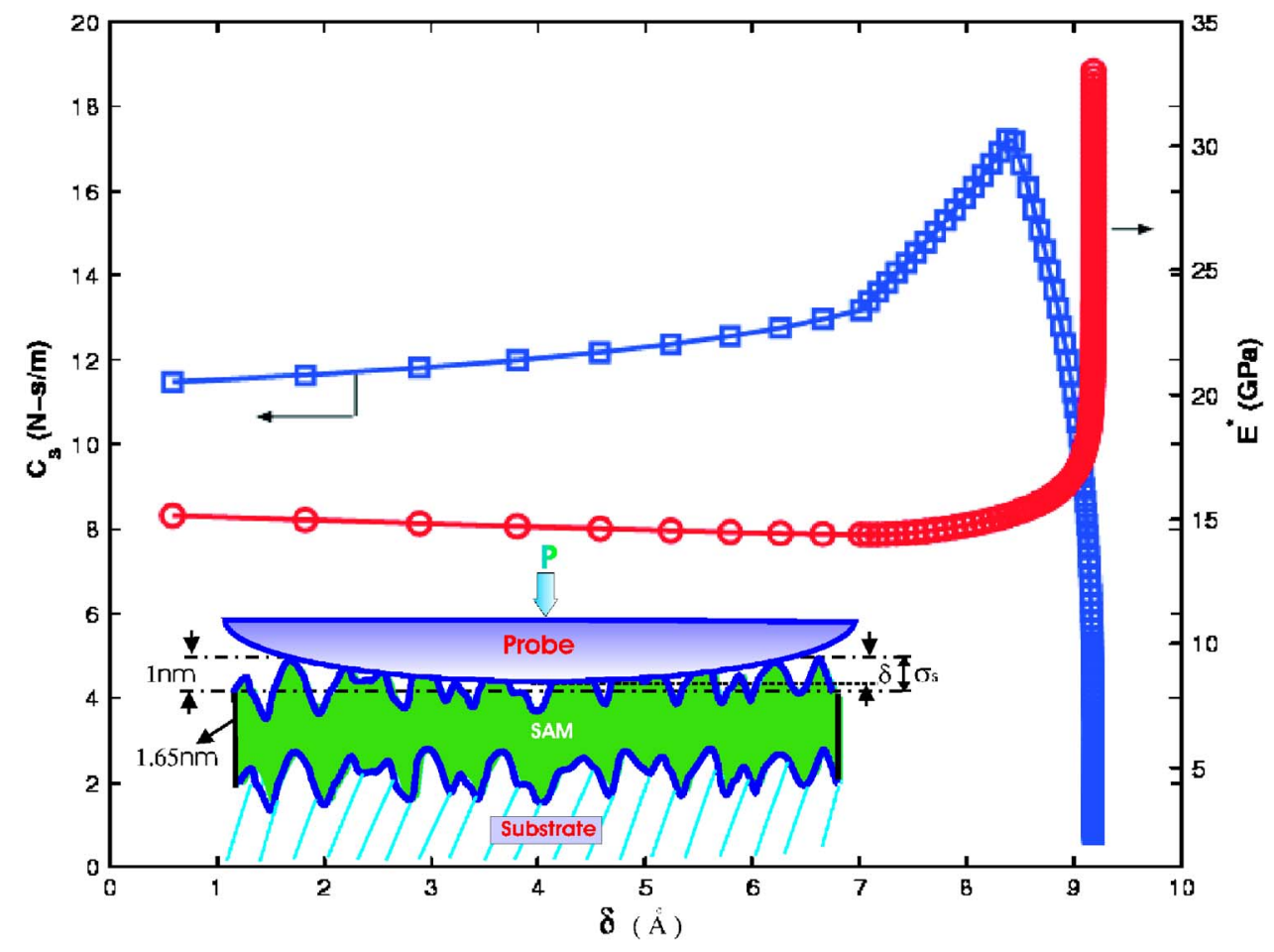

FIG. 3. (Color online) Damping constant $\left(C_{s}\right)$ and Young's modulus $\left(E^{*}\right)$ of FOTS SAM as a function of indented displacement $\delta$ (shown as a schematic in inset).

ecule as the terminal end of different molecules assembled on a rough surface comes into contact at different levels of probe displacement (see inset of Fig. 3). Methodologically, as long as one or both of the contacting surfaces is geometrically curved, the information generated is aggregate even if the surface of the probe and the surface are made atomistically smooth. We concede that if the surfaces are atomistically smooth the scatter in experimental data will be reduced considerably but use of such surfaces takes us away from lubrication which is the motivation for the work.

\section{DISCUSSION: A PHYSICAL EXPLANATION}

With the help of some existing molecular dynamics simulations ${ }^{16,23,25-27}$ of compression of self-assembled monolayers we attempt a physical understanding of the results reported here. When a monolayer is compressed by spherical tip of large radius $(1.12 \mathrm{~mm})$ two principal modes of motion are possible. (1) The molecules (chains) are compressed collectively against each other [Fig. 4(a) schematically exemplifies the mode] and (2) bonds in individual molecules rotate, twist, bend, and kink to yield a "defective" molecule. These defects are generically termed gauche defects. ${ }^{13}$ The two modes are not totally independent of each other as changes in intermolecular distance by compression may also generate gauche defects in certain situations. ${ }^{25}$

Figure 4(b) shows a highly idealized schematic of polymer chains in a semi-infinite half space being pushed away by indentation. With indentation the chains translate radially
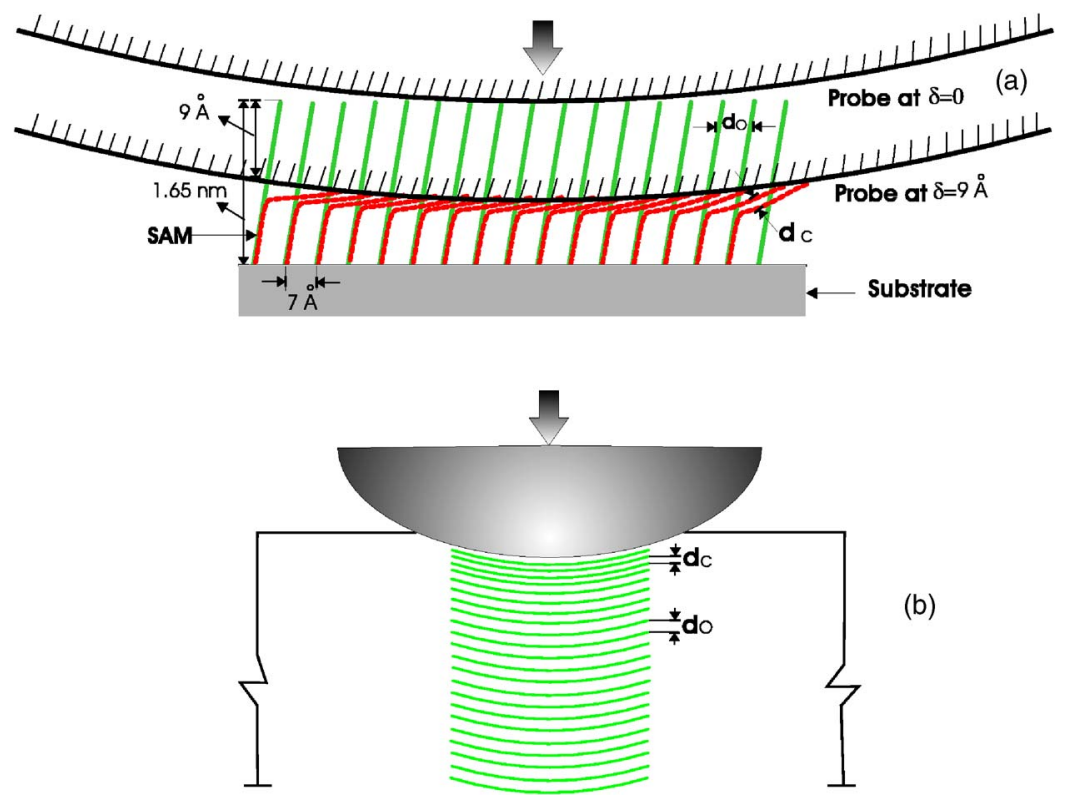

(b)
FIG. 4. (Color online) (a) Schematic showing an envisaged deformation of self-assembled monolayer being compressed by a spherical probe. $d_{c}$ is the intermolecular separation, $d_{c}$ $<d_{0}$ where $d_{0}$ is the van der Waals equilibrium distance between adjacent molecular chains. (b) Schematic showing the compression of idealized polymer chains in a semi-infinite space. In the far field the equilibrium separation is recovered. 
out to relax stresses yielding a strain distribution $\left[\left(\varepsilon_{r}\right)_{r}\right.$ $\approx\left[d_{0}-\left(d_{c}\right)_{r}\right] / d_{0} \propto 1 / r^{n}$, where $n$ is $2-3$ and $r$ is the radial distance] which does not change substantially with penetration of the probe into the medium and in the far field $r \rightarrow \infty$, $d_{c} \rightarrow d_{0}$, the (van der Waals) equilibrium interchain distance. The situation changes dramatically when the chains are anchored to the substrate. The stresses cannot relax as in the case of indentation into a semi-infinite space and all the adjacent chains pinned in the gap between the indentor and the substrate continue to become compressed against each other [Fig. 4(a)], reducing the interchain distance $\left(d_{c}<d_{0}\right)$ with increasing penetration. This reduction accommodates the volume displaced by the indentor. We note at this stage that interchain repulsion supports the applied indentation load and going by the expected repulsion potential ${ }^{8}[w(d)$ $\propto A / d^{12}$, where $A$ is a constant] a large increase in force is needed to make a very small difference in the interchain or interparticle distance. Thus for the self-assembled monolayer when the penetration is sufficiently advanced the indented material becomes very stiff (stiffness $S_{v} \equiv d P / d v$, where $v$ is the volume) as large increases in indentation load are necessary to give rise to a small change in volume per chain. This has been demonstrated explicitly by molecular dynamics (MD) simulation of a self-assembled monolayer being compressed between flats. ${ }^{26}$ The present experimental configuration of compression by a large-radius indentor approaches the flat-plate compression configuration used in the above MD simulation more than it resembles the sharp tip experimental configuration of the AFM. ${ }^{13}$ To explore this mechanism as one of relevance to our experimental results we first determine the spatial density of the SAM using the AFM, Fig. 5 (Thermomicroscope Explorer, Veeco Instruments, USA). Knowing this density and the contact area-applied load characteristics deconvoluted from our contact mechanical calculations [Eq. (3)] we determine the number of molecules under contact as a function of applied load as shown in Fig. 6. Figure 7 shows the volume per chain as a function of load to be very similar to that calculated using molecular dynamics simulation. ${ }^{26}$ In the present case the flexible platform used to support the SAM substrate limits the maximum work done on the SAM to about $18 \mathrm{~kJ} / \mathrm{mol}$ as seen in Fig. 8 . The SAM therefore never reaches the desorption state [(130 kJ/mol) (Ref. 16)] and unlike what has been demonstrated in AFM experiments ${ }^{15,26,27}$ is intact even at the highest load used. The only direct validation that can be made of our experimental results is a comparison with the molecular dynamics simulation of flat-plate compression of an alkane thiol SAM, ${ }^{31}$ which reports an increase of about $1 \mathrm{eV} /$ chain for a $3 \AA$ compression immediately after contact. Our experimental results (Fig. 8) give about $0.3 \mathrm{eV} /$ chain for a similar degree of compression. Another MD simulation of friction in an alkane thiol slid between flat plates of diamond shows a level of about $0.14 \mathrm{eV} /$ chain at the commencement of sliding. ${ }^{26}$

A reduction in interchain distance and concomitant increase in repulsion with indentation supports the indentation load as the indentor approaches the substrate. This mode of chain movement leads to a material resistance to applied load which should increase with indentor displacement. This ten-

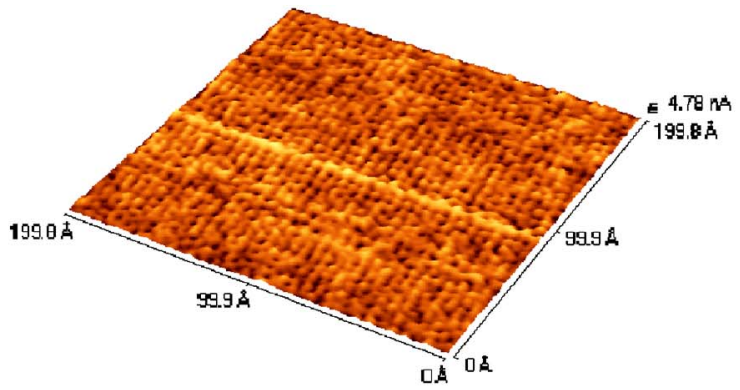

(a)

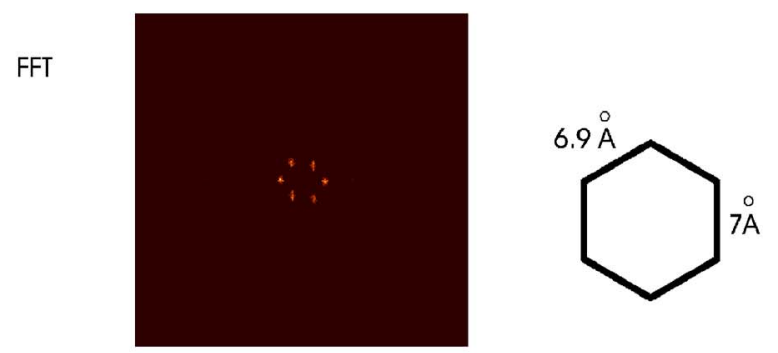

(b)

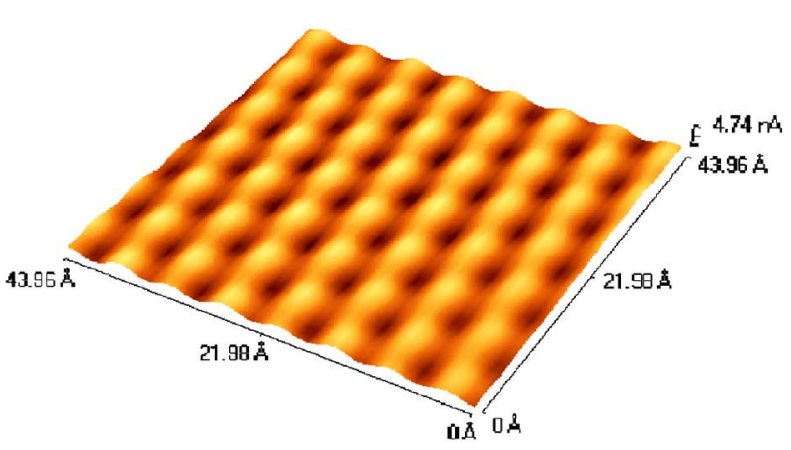

(c)

FIG. 5. (Color online) Atomic force image of $1 \mathrm{H}, 1 \mathrm{H}, 2 \mathrm{H}, 2 \mathrm{H}-$ perfluorooctylsilane monolayer self-assembled on aluminum. (a) Friction force image of raw data; (b) fast Fourier transform image of (a) showing a hexagonal lattice; (c) filtered friction force image.

dency, we argue, is opposed by the other principal mode of molecular motion related to the generation of gauche defects. When the indentor comes into contact with the monolayer the gauche defects at the top of the molecule where there exists a large degree of freedom of movement are generated easily and rapidly. The upper reaches of the molecules become disordered and amorphous, exhibiting a liquidlike response to force where applied stresses relax quickly (small relaxation time). This has been demonstrated experimentally ${ }^{13}$ and using molecular dynamics simulation. ${ }^{27}$ The hinterland of a chain, however, remains relatively undisturbed, the structural order increasing in moving down the backbone from the surface. With penetration gauche defects are generated even in the hinterland but with the expenditure of much energy. Gauche defects in general are known to provide channels of energy dissipation. ${ }^{9,28}$ Coming back to our experimental procedure, we apply low-frequency sinusoidal oscillation to the indentor as it penetrates the monolayer and record the gain and phase shift $(\Delta \phi)$ of the signal. 


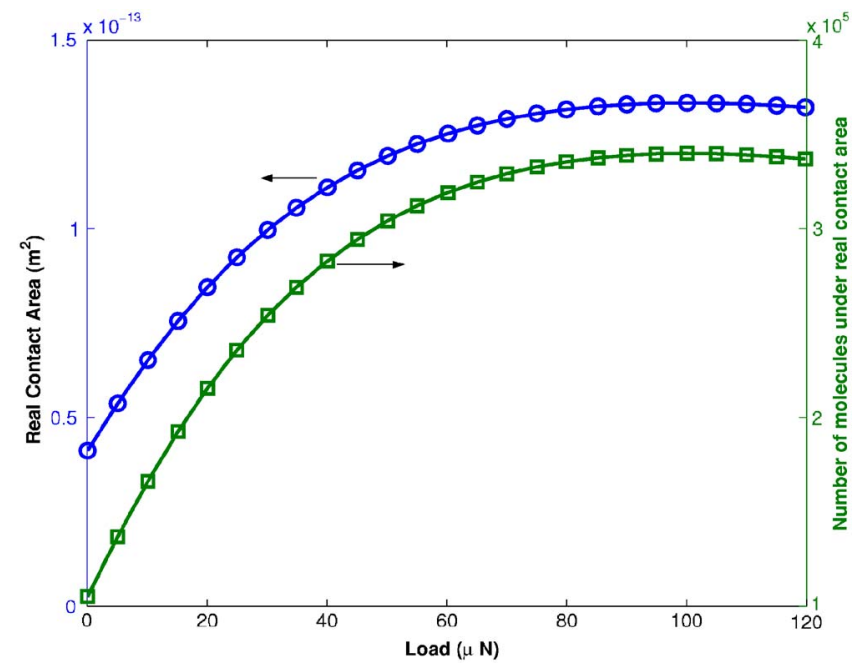

FIG. 6. (Color online) Real area of contact (probe radius $=1.12 \mathrm{~mm}$ ) and number of molecules under the real area of contact in compression of FOTS, estimated using Eq. (3) and Fig. 5.

From these data using the Maxwell model of viscoelastic material, we can estimate ${ }^{29}$ the relaxation time $\tau_{r}=\left(C_{S}\right.$ $\left.+K_{s}^{2} / \omega^{2} C_{s}\right) /\left(K_{s}+\omega^{2} C_{s}^{2} / K_{s}\right)$, where $\omega$ is the drive frequency and $C_{s}$ and $K_{s}$ are given by Eq. (1). The relaxation time is proportional to the effective viscosity of a viscoelastic body. Figure 9 shows the variation of $\tau_{r}$, the relaxation time and that of the retardation time $\tau_{d}\left(=C_{s} / K_{s}\right)$ estimated using the Kelvin model, with load and penetration into the molecule. As channels of energy dissipation become available with the creation of gauche defects and it is possible to dissipate the energy by motion such as torsion at the molecular level, it becomes possible for the molecules to relax the imposed stresses quickly and easily. $\tau_{r}$, which is an indicator of the viscous response, is somewhat insensitive initially to increasing load. This trend is in agreement with those reported by Demirel and Granick ${ }^{11}$ and Jeffrey et al. ${ }^{29}$ Increasing load is known $^{27}$ to increase gauche defects and ease of energy dis-

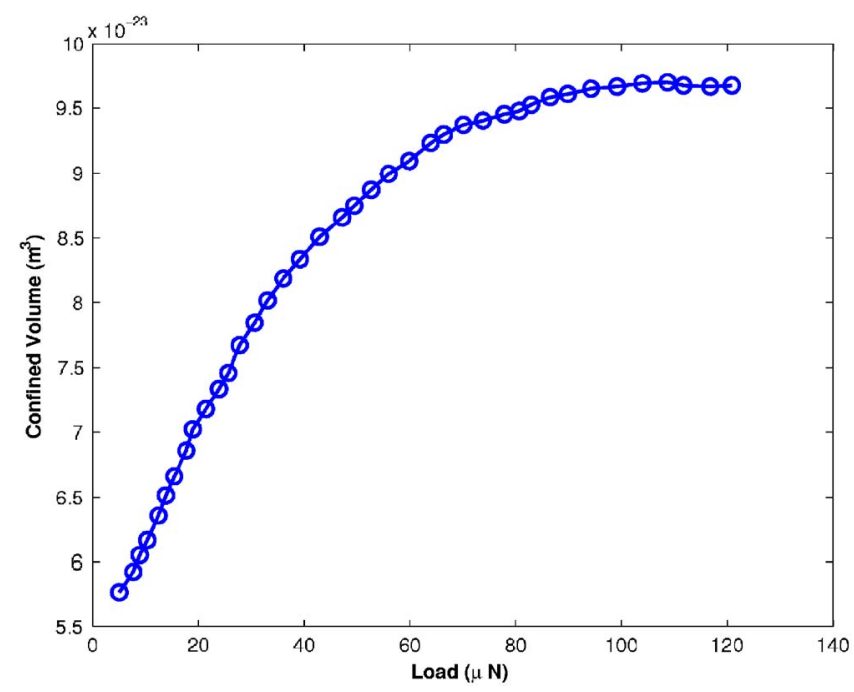

FIG. 7. (Color online) Estimated confined volume under the real area of contact as a function of applied load.

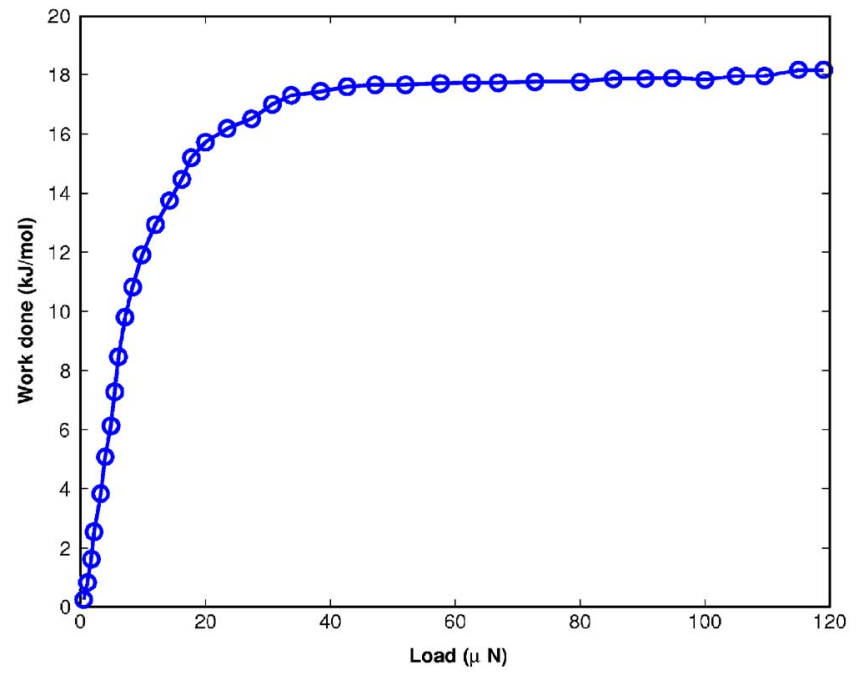

FIG. 8. (Color online) Work done on the system per mole by the probe, estimated knowing the load-displacement characteristics (Figs. 1 and 3) and the volume under real contact area (Fig. 7).

sipation in self-assembled monolayers. Simultaneously the interchain distance continues to be reduced and the volume trapped between the indentor and the substrate becomes more and more constrained, reducing the freedom of motion of individual molecules. Figure 9 shows that there is a critical load $(20 \mu \mathrm{N})$ at which there is an inflection in the stiffness-load characteristic and a sharp increase in $\tau_{r}$, the relaxation time. We believe that this load marks a phase transition from a viscous liquidlike to a solidlike response of the molecule to applied force. At high loads beyond this transition, by now the highly constrained volume trapped between the indentor and the substrate suppresses free rotation and other motions of single molecules and does not allow the applied stress to relax. The relaxation time tends to $10^{2} \mathrm{~s}$. Response to force is no longer related to dissipative motions of individual molecules but to that of a collective block of a near rigid solid consisting of immobile molecules. Such phase transitions have been experimentally observed, albeit more gradually, for spherical hydrophobic molecules on a

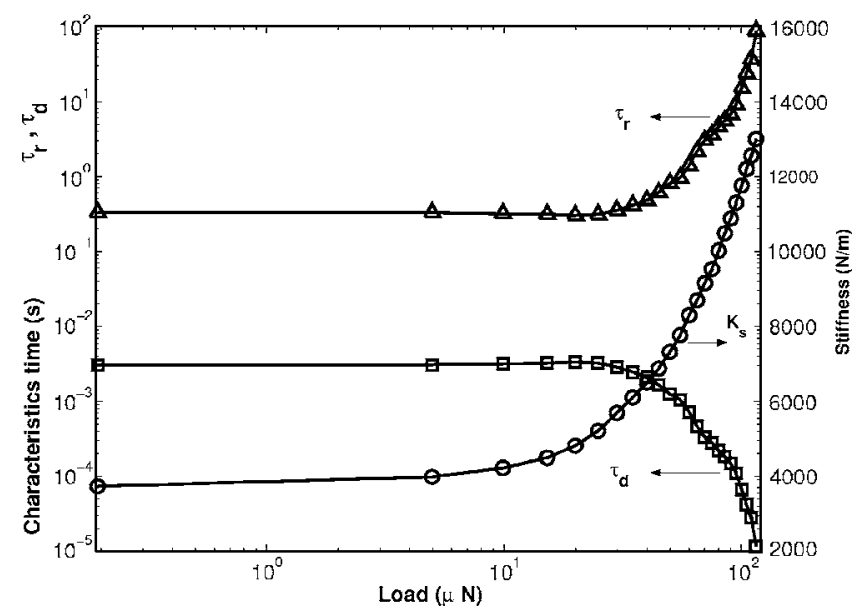

FIG. 9. The relaxation time $\tau_{r}$, the retardation time $\tau_{d}$, and the stiffness of FOTS SAM as a function of applied load. 
substrate as a function of driving frequency. ${ }^{11}$ Molecular dynamics simulation of friction has demonstrated ${ }^{26}$ the disappearance of large-amplitude periodic variation in friction force in tightly packed molecules in alkane monolayers due to the large physical constraint imposed on the trapped volume at high loads. Joyce et al. ${ }^{15}$ have also observed a large increase in stiffness at a critical load in compressing selfassembled monolayers and have argued this to be a substrate effect as, for example, is observed in nanoindentation of thin solid films. Our work here contests such an explanation and argues that the dramatic increase in stiffness and modulus observed at a critical indentation load is due to a phase transition in the monolayer from a liquidlike state to a solidlike state.

To validate our findings against previous experimental work is not easy as experimental objectives and methods used previously vary widely and are in some cases quite different from ours. For similar molecules the stiffness we report $\left(4 \times 10^{3} \mathrm{~N} / \mathrm{m}\right)$ at the commencement of contact is similar to that reported for octamethylcyclotetrasiloxane $(\text { OMCTS })^{11}\left(3.2 \times 10^{3} \mathrm{~N} / \mathrm{m}\right)$ and for alkane thiol $\left(10^{3}\right.$ $\mathrm{N} / \mathrm{m}) .{ }^{15}$ Our experimental methods are very similar to those of Jeffery et al. ${ }^{29}$ and the trends for $\tau_{r}$ and $\tau_{d}$ that they report are also similar to what we observe here (Fig. 9) but absolute values elude comparison as their test molecule is water of very low stiffness $(<0.1 \mathrm{~N} / \mathrm{m})$. The work of Demirel and Granick $^{11}$ is concerned with finding the relaxation time at the liquid-to-solid phase transition at different levels of confinement by varying the driving frequency. The relaxation time $\left(10^{1}-10^{3} \mathrm{~s}\right)$ they deconvolute for OMCTS at low frequency and high confinement bears comparison with our stressinduced transition at low frequency.

Now moving back to our engineering concern of load bearing by the monolayer, the modulus of elasticity data shown in Fig. 3 exhibits two distinct regimes, (I) where the modulus decreases slightly with probe intervention ( $d$ $<7 \AA$ ) and (II) where the modulus increases steeply with probe intervention in the $d>8.5 \AA$ regime. We may associate the two regimes with a weak and a stiff spring acting in series and representing the response of the upper end and the lower part of the molecular backbone. The regime I response is dominated by the disordered upper part of the molecule. There is significant displacement in this regime as the probe penetrates a soft layer supported by the stiff elastic hinterland. The aggregate disorder increases with penetration as there is a gentle fall in the modulus, the modulus varying only slightly between 15 and $14.5 \mathrm{GPa}$.

In regime II the damping reduces to nil with a small increase in displacement. This implies that the probe is now penetrating an ordered crystalline material. The response here is that of a nonlinear elastic spring. The displacement and contact area change little with load in this regime. This implies that work is done by external load not to deform significantly but to overcome increasing resistance of the SAM to applied force as the probe penetrates into the SAM. Increasing applied load is now supported by increasing repulsion between the molecules as the separation between them is attempted to be reduced.

\section{CONCLUSION}

What is unusual about the work reported here is that the choice of a flexible platform supporting the monolayer allowed us to control the mean contact pressure, obtain high resolution, and limit penetration into the monolayer to about midlength of the molecular backbone as well as limit the energy input to the monolayer to a modest level. Our maximum pressure of $\approx 1 \mathrm{GPa}$ is significantly lower than those used by others to press monolayers; AFM experimental $\equiv 30 \mathrm{GPa},{ }^{15} \mathrm{AFM}$ simulation $\equiv 32 \mathrm{GPa},{ }^{30,31}$ and flat plate compression simulation $\equiv 60 \mathrm{GPa} .{ }^{26} \mathrm{We}$ are thus able to access the monolayer about midlength and the work we do on the monolayer, maximum $\equiv 18 \mathrm{~kJ} / \mathrm{mol}$, is therefore not destructive. The maximum work input values reported by Joyce et al. ${ }^{15}(150 \mathrm{~kJ} / \mathrm{mol})$, Tupper and $\operatorname{Brenner}^{31}(300 \mathrm{~kJ} / \mathrm{mol})$, and Tutein et al. ${ }^{25}(300 \mathrm{~kJ} / \mathrm{mol})$ are well above the chemisorption energy ${ }^{26}$ of $130 \mathrm{~kJ} / \mathrm{mol}$. We admit that uncertainty exists regarding the absolute values of the penetration we report due to the rough nature of the surfaces and the rather simple contact mechanical analysis (where the modulus is insensitive to load) we have used. A more rigorous calculation brings the (transition) penetration level to about $6-7 \AA$ and the maximum work done to about $30-45 \mathrm{~kJ} / \mathrm{mol}$. This, however, does not change the inference we draw from the work that there is a phase transition which occurs at a mean pressure of about $\approx 0.2 \mathrm{GPa}$, where the viscous liquidlike behavior of the monolayer under compression changes to a more solidlike behavior indicated by high relaxation time constant. We believe that the subtle change we detect at a low mean pressure may not be as well defined and therefore as easily detectable if the experiment were done in a mean pressure range which is $30-50$ times that used here.

\section{ACKNOWLEDGMENTS}

We are grateful to DRDO (India), Centre for High Technology (Ministry of Petroleum, Govt. of India), and General Motors (R\&D), Warren, U.S., for grants which made this work possible.

\footnotetext{
*Corresponding author. Electronic address: skbis Microelectromech. Syst. 7, 252 (1998).

@ mecheng.iisc.ernet.in $\quad{ }^{3}$ Ian C. Clarke et al., Wear 250, 188 (2001).

${ }^{1}$ F. P. Bowden and D. Tabor, The Friction and Lubrication of ${ }^{4}$ H. Spikes, New Dir. Tribol., Plenary Invited Pap. World Tribol. Solids (Oxford University Press, Oxford, 1950), Part I.

${ }^{2}$ U. Srinivasan, M. R. Houston, R. T. Howe, and R. Maboudian, J.

Congr., 1st 1997, 355 (1997).

${ }^{5}$ B. Bhusan, Modern Tribology Handbook (CRC Press, Washing-
} 
ton, D.C, 2001), Vols. I and II.

${ }^{6}$ M. A. Wimmer, C. Sprecher, R. Hauert, G. Täger, and A. Fischer, Wear 255, 1007 (2003).

${ }^{7}$ T. Murakami, Y. Sawae, K. Nakashima, and J. Fisher, in Thinning Films and Tribological Interfaces (Proceedings of the 26th Leeds-Lyon Symposium on Tribology), edited by D. Dowson et al. (Elsevier Science B.V., Amsterdam, 2000), p. 317.

${ }^{8}$ J. N. Israelachvilli, Intermolecular and Surface Forces, 2nd ed. (Acadamic Press, New York, 1992).

${ }^{9}$ J. Peachey, J. Van Alsten, and S. Granick, Rev. Sci. Instrum. 62, 643 (1991)

${ }^{10} \mathrm{~J}$. N. Israelachvili and A. D. Berman, in Handbook of Micro/Nano Tribology, edited by B. Bhushan (CRC Press, New York, 1999), pp. 371-482.

${ }^{11}$ A. L. Demirel and S. Granick, Phys. Rev. Lett. 77, 2261 (1996).

${ }^{12}$ Q. Du, X.-d. Xiao, D. Charych, F. Wolf, P. Frantz, Y. R. Shen, and M. Salmeron, Phys. Rev. B 51, 7456 (1995).

${ }^{13}$ R. W. Carpick and M. Salmeron, Chem. Rev. (Washington, D.C.) 97, 1163 (1997).

${ }^{14}$ R. A. Quon, A. Ulman, and T. K. Vanderlick, Langmuir 16, 3797 (2000).

${ }^{15}$ S. A. Joyce, R. C. Thomas, J. E. Houston, T. A. Michalske, and R. M. Crooks, Phys. Rev. Lett. 68, 2790 (1992).

${ }^{16}$ J. I. Siepmann and I. R. McDonald, Phys. Rev. Lett. 70, 453 (1993).

${ }^{17}$ D. Devaprakasam, S. Sampath, and S. K. Biswas, Langmuir 20, 1329 (2004).

${ }^{18}$ G. Luengo, F-J. Schmitt, R. Hill, and J. N. Israelachvili, Macromolecules 30, 2482 (1997).

${ }^{19}$ D. Devaprakasam and S. K. Biswas, Rev. Sci. Instrum. 74, 1228
(2003).

${ }^{20}$ D. Devaprakasam and S. K. Biswas, Rev. Sci. Instrum. 76, 035102 (2005).

${ }^{21}$ B. N. Lucas, C. T. Rosenmeyer, and W. C. Oliver (unpublished).

${ }^{22}$ D. Maugis, Contact, Adhesion and Rupture of Elastic Solids (Springer, Berlin, 1999). Overload calculation: $P_{a d}=2 \pi \omega r_{a} n$, where $r_{a}$ is the asperity radius $\approx 10^{-6} \mathrm{~m}$, work of adhesion $\omega$ $=10 \mathrm{~mJ} / \mathrm{m}^{2}$, obtained from the pull-off force (Fig. 1 inset) taking account of roughness, and $n$ is the number of summits in contact $\approx 300$ at $P=120 \mu \mathrm{N}$. The calculation gives $P_{a d}=6.2$ and $17 \mu \mathrm{N}$ at $P=20$ and $120 \mu \mathrm{N}$, respectively. If $P_{t}=P+P_{a d}$, the error in $P_{t}$ estimate in assuming $P_{a d}$ to be constant in the $20-120 \mu \mathrm{N}$ load range is $8 \%$.

${ }^{23}$ Y. Leng and S. Jiang, J. Chem. Phys. 113, 8800 (2000).

${ }^{24}$ D. K. Owens and R. C. Wendt, J. Appl. Polym. Sci. 13, 1741 (1969).

${ }^{25}$ A. B. Tutein, S. J. Stuart, and J. A. Harrison, Langmuir 16, 291 (2000).

${ }^{26}$ P. T. Mikulski and J. A. Harrison, J. Am. Chem. Soc. 123, 6873 (2001).

${ }^{27}$ A. B. Tutein, S. J. Staurt, and J. A. Harrison, J. Phys. Chem. B 103, 11357 (1999).

${ }^{28}$ N. D. Shinn, T. M. Mayer, and T. A. Michalske, Tribol. Lett. 7, 67 (1999).

${ }^{29}$ S. Jeffery, P. M. Hoffmann, J. B. Pethica, C. Ramanujan, H. O. Ozer, and A. Oral, Phys. Rev. B 70, 054114 (2004).

${ }^{30}$ M. Hartig, L. F. Chi, X. D. Liu, and H. Fuchs, Thin Solid Films 327-329, 262 (1998).

${ }^{31}$ K. J. Tupper and D. W. Brenner, Langmuir 10, 2335 (1994). 